\title{
On the Influence of Original Family on Individual Mental Health — From the Family System Theory
}

\author{
Niu Hui \\ Department of Social Work, Northeast forestry university, Grammar school, Harbin, The Heilongjiang River, China \\ 1940154316@qq.com
}

\begin{abstract}
Native families at the individual initial growth plays an important role in the process of development, family interactions, family structure, family atmosphere and parents upbringing of their children, intimate degree will of individual behavior model, the expression of emotion, to interact with the opposite sex, and other aspects have a major impact. Family system theory is different from individual psychotherapy theory. It is a treatment model that treats the whole family as a treatment unit. It aims to solve individual problems by analyzing the interaction and communication between the whole family members. Therefore, family system theory is one of the important met-hods to understand the causes of individual psychological behavior problems. From t-he perspective of family system theory, this paper analyzes the influence factors of family of origin on individual psychology, and explores ways to improve individual mental health.
\end{abstract}

Keywords: original family, family system theory, individual mental health

\section{浅谈原生家庭对个体心理健康的影响 从家庭系统理论视角出发}

\author{
牛慧
}

东北林业大学社会工作专业, 哈尔滨, 黑龙江, 中国

1940154316@qq.com

\section{摘要}

原生家庭在个体最初的成长发育过程中扮演着重要的角色, 家庭成员的交往方式, 家庭结构, 家庭氛 围以及父母对子女的教养方式、亲密程度都会对个体的行为模式, 情绪情感的表达, 与异性的互动方 式等多个方面产生重要的影响。家庭系统理论不同于个别心理治疗理论, 它是一种把整个家庭作为治 疗单位的治疗模式，通过分析整个家庭成员之间的互动和沟通模式，以达到解决个体问题的目的。因 此家庭系统理论也是了解个体心理行为问题产生原因的重要方法之一。本文从家庭系统理论视角出 发，分析原生家庭对个体心理的影响因素，探究提高个体心理健康水平的方法。

关键词: 原生家庭, 家庭系统理论, 个体心理健康

\section{1. 前言}

大的那个家庭称为原生家庭 [1]。从现有的资料和调查 来看, 原生家庭对于个体而言, 不论在人格形成还是心 理健康方面都有着重要影响。

每个人刚出生时都犹如一张白纸, 经过身处各种环 境的影响, 逐渐过渡到一个 “社会的人”。而家庭正是 一个人一生中最初接触并生长的环境, 一个人性格的塑 造、自我意识的培养、人生观价值观的形成, 以及他与 他人交往时的情感、态度表现等都与其原生家庭有着错 综复杂的联系。通常意义上, 我们把一个人从小出生长

\section{2. 家庭系统理论概述}

家庭系统理论泛指在家庭心理学中运用系统思维, 对个体、夫妻和家人在相互关系中以及活动环境中的情 感、思想和行为进行研究的家庭心理学理论 [2]。它是 
由美国心理治疗专家默里・鲍恩（Murray Bowen）教授 在 20 世纪 40 年代末提出, 当时心理治疗强调人的独 特意识和其本能冲动, 避免治疗者与患者家属接触。但 鲍恩这样并不能很好的理解患者行为, 他在一些治疗过 程中与患者和他的家属尤其是父母之间, 很容易产生情 绪的相互影响。在研究过程中, 鲍恩把家庭看成一个情 绪单位, 发现家庭成员之间的活动是相互作用的, 因此 Murray Bowen 提出了家庭系统理论, 之后, 他的助手 Michael E. Kerr 完善了该理论。

张志学用两个变量和一个核心概念加以说明家庭 系统理论。两个变量分别是自我分化(differentiation of self)和慢性焦虑(chronic anxiety), 一个核心概念就是三 角(triangle)。自我分化指的是个体从早期依赖的成长环 境中逐渐成长为一个独立个体的过程。它与群聚对应, 一个自我分化良好的个体应该是既能和他人产生良好 的情绪和行为的互动, 又能拥有良好的自我意识, 能独 立思考和行动。在一个分化不良的家庭中, 个体的独立 性和自我意识都发展得较差, 他们与家庭的情绪卷入很 深, 家庭成员的情绪很容易影响个体的情绪。慢性焦虑 是指人对于想象中的威胁的一种反应, 是由于人们害怕 可能发生的事而产生的。慢性焦虑的产生原因主要是个 体所在的关系系统受到干扰, 家庭关系的平衡遭到破 坏, 研究表明, 慢性焦虑主要是人在后天习得的, 尤其 是在从小生长的家庭环境中 [3]。其中, 自我分化与慢 性焦虑有着一定负相关性, 基本分化水平越低, 慢性焦 虑水平则越高。而三角这个概念是描述三人关系系统的 动力模式。在这种模式中, 关系的变化是由慢性焦虑决 定的。在人际交往中, 如果没有什么外来的压力, 人们 的焦虑较小, 两人之间的关系通常就是稳定的。但是当 人们的焦虑增加时, 常常会有第三个人参与到原先的两 人关系中, 由此构成了一个三角。

\section{3. 原生家庭影响个体心理健康的主要因素}

根据国内外现有的研究表明, 关于原生家庭对个体 的心理健康的影响因素主要包括以下两个方面。

\section{1 父母的婚姻关系}

家庭中父母的婚姻状况、亲密程度对个体心理健康 产生重要影响。Murray Bowen 的家庭系统理论认为, 个体原生家庭中父母的关系模式会影响他未来的人际 关系甚至是亲密关系。人们倾向于在一段亲密关系中表 现出与自己早期原生家庭中家庭成员相似的关系模式 [4]。

如果父母在经营婚姻时得当, 相濡以沫, 则子女则 可以在观察中学会与异性相处的方式, 树立正确的恋爱 婚姻观。Murray Bowen 认为, 人倾向于选择那些与自 己的分化水平相当的人作为自己的另一半来组建新的 家庭, 因此他们建立的家庭与自己的原生家庭有着很大 的相似性。个体的原生家庭通过慢性焦虑和三角关系来
影响个体的分化水平, 如果在新的家庭中, 夫妻双方中 的一方或者双方分化水平较低, 原生家庭的影响就逐渐 展现出来。这些原生家庭的代际影响对个体的价值观、 相处模式、情感表达、教育下一代的方式等都起着不容 小觑的作用。大量的研究已验证了这一点：原生家庭特 征能够有效预测其后代的婚姻质量和婚姻满意度 [5]。 同时, 如果夫妻双方的婚姻出现问题, 家庭关系紧张, 甚至婚姻破裂、离异, 并且未及时弥补不良的婚姻关系 对子女的影响, 便可能会导致子女出现叛逆、性格孤僻、 拒绝与异性接触等。一项关于原生家庭对个体婚姻的干 涉与支持的研究调查也显示, 个体对原生家庭支持的感 知能有效正向预测其自身的婚姻质量，而对原生家庭干 涉的感知能显著负向预测其自身与配偶的婚姻质量 [6]。

\section{2 亲子关系}

亲子关系是个体一生中最早接触到的关系, 与家庭 中的其他人际关系相比, 亲子关系是对个体心理健康影 响最深。有关研究显示, 个体所在家庭中的亲子关系好 坏影响个体的人际交往方式。在婚姻关系对儿童社会情 绪适应的影响研究中, 亲子关系被认为起到了中介作 用, 即父母婚姻关系不良会引起亲子之间的敌 对关系, 进而导致儿童出现各种适应障碍和问题行为。父母婚姻 关系不和谐的家庭会随着儿童成长进而出现许多不良 适应的问题, 而婚姻关系和谐的家庭, 儿童的社会情绪 适应良好 [7]。因此, 父母良好的婚姻关系有助于形成 安全、亲密的亲子关系，而家庭气氛紧张、夫妻婚姻关 系不融洽则不能带给孩子应有的安全感，从而造成疏 远、冷漠、紧张的亲子关系。家庭中高质量的婚姻关系 不仅与当前父母与子女高质量的亲子关系有显著的相 关，还能预测两年后高质量的亲子关系 [8]。所以，原 生家庭中亲子关系的好坏，对个体的影响深远。

同时, 原生家庭中父母所采取的教养方式, 也会影 响亲子关系。理解宽容的教养方式更有利于培养良好的 亲子关系, 进而减少亲子间发生矛盾与冲突的强度与次 数, 营造出一个和睦的家庭环境, 推动个体发展形成积 极乐观的心理; 而严格独断的家庭教养方式则可能加剧 亲子冲突, 使个体产生偏激的想法或行为。也有研究围 绕亲子冲突最频发的青春期展开, 认为这时的个体自主 意识增强, 父母

需要根据实际情况适当调整自己的教养方式[9]。 如果父母采取越严厉、惩罚越多的教养方式, 青少年所 产生的逆反心理就越强。所以与采取民主型教养方式相 比, 那些在专制型教养方式下成长的青少年逆反心理会 更为严重 $[10]$ 。青春期个体的心理健康状态对个体未来 的生理或心理发展都起着决定性作用。因此, 原生家庭 教养方式对个体的心理发展也至关重要。 


\section{4. 小结}

家庭系统理论为分析和治疗个体心理问题提供了 一个特殊的切入点, 使得心理治疗脱离于简单地对个体 问题的分析而立足于原生家庭这一完整的系统, 从而在 家庭成员的互动中寻找个体行为、情感与思维的根本原 因。另外, 大量的研究也让人们意识到原生家庭对个体 心理与行为健康发展的重要性。学校教育者常说: “每 一个问题孩子背后, 往往都有一个问题家庭。” 个体成 长的过程, 就是自我感情从其情感所依附和寄托的家庭 系统中分化出来的过程, 自我分化是个体成熟和心理健 康的最关键个性变量 [1]。因此, 个体要直视原生家庭 中的不完美, 勇于面对原生家庭对自身的影响, 并不断 完善自我, 以促进个体心理健康发展。同时, 当个体在 处理婚姻关系时, 应该努力提高自我分化水平, 减少原 生家庭中的婚姻关系对子女发展产生过多的影响。并且 在处理亲子关系时, 要选择适当的教养方式, 培养良好 的亲子关系, 为子女的健康发展提供一个好的家庭氛 围。

\section{REFERENCES}

[1] Lu jing, Cao lili. Analysis on the influence of family of origin from the perspective of chaos theory $[J]$. Journal of Qiqihar university: philosophy and social sciences, 2011 (1): 71-74.

[2] Zhang zhi-xue. Development and current situation of family system theory [J]. Psychological research, 1990 (1).

[3] Michael E. Kerr. Chronic Anxiety and Defining a Self [J]. The Atlantic Monthly, 1988(9):35.

[4] Lu jing, Cao lili. Analysis on the influence of family of origin from the perspective of chaos theory [J]. Journal of Qiqihar university: philosophy and social sciences, 2011 (1): 71-74.

[5] W.K. Halford, M. R Sanders, B. C Behrens. Repeating the Errors of Our Parents? Family-of-Origin Spouse Violence and Observed Conflict Management in Engaged Couple[J]. Family Process, 2000, 39(2):219-235.

[6] Yuan xiaojiao, fang xiaoyi. [C] // Chinese psychological society. Improvement of psychology and innovation ability -- proceedings of the 16th national psychology academic conference. Nanjing: Chinese psychological society, 2013.

[7] Wu yuwei. On the influence of family of origin on individual development -- from the perspective of family system theory [J]. Journal of quanzhou normal university.

[8] Shek D. T. L. Parental Marital Quality and Well-being, Parent-child Relational Quality, and Chinese Adolescent Adjustment[J]. American Journal of Family Therapy, 2000, 28(2): 147-162.

[9] Cao Jun. Analysis of relevant factors of adolescent rebellious psychology [D]. Nanjing: Nanjing medical university, 2008.

[10] Qiu Wenting. Research on the relationship between middle school students' rebellious psychology and their parents' parenting style [D]. Chongqing: southwest university, 2009. 